\title{
FREQUENCY RESPONSE PREDICTION FOR ROBOT ASSISTED MACHINING
}

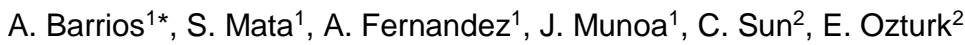 \\ ${ }^{1}$ IDEKO, Dynamics and Control, Elgoibar, Spain \\ ${ }^{2} \mathrm{AMRC}$ with Boeing, The University of Sheffield, Sheffield, UK \\ *Corresponding author; e-mail: abarrios@ideko.es
}

\begin{abstract}
Robotics is increasing its presence in the machine tool sector. One interesting application for robot assisted machining involves a robot locally increasing the stiffness of a thin walled part to suppress regenerative vibrations and minimize part deformations during machining.

Simulating the dynamics improvement achieved when coupling the robot and the part is of high concern, in order to guarantee the appropriate performance of the assisted machining. Receptance Coupling Substructure Analysis (RCSA) technique for High Speed Machining (HSM) dynamics simulation has been expanded to derive the frequency response of the assembled system composed by the coupling of the thin walled part and the robot.
\end{abstract}

\section{Keywords:}

Robot; Dynamics; Frequency response; Receptance coupling; Machining

\section{INTRODUCTION}

Robotics is revolutionizing manufacturing as robots become smarter, faster and cheaper, increasing its presence for high value tasks rather than traditional repetitive or dangerous tasks [Pricewaterhouse 2014].

Machining of thin walled parts is a challenging manufacturing process since the low stiffness of thin walled parts leads to undesirable vibrations and deformations of the part, due to instability in the machining process, causing a negative effect on machining accuracy and surface quality of the final part.

Traditionally milling stability theory [Altintas 2012] is applied to eliminate vibrations and increase productivity by tuning process parameters. However, occasionally additional actions are required to modify the dynamics of the part and obtain a stable machining operation. Acting on the fixturing of the part provides a considerable effect on the improvement of the dynamics of the part.

Usually the part is clamped to the table at a discrete number of predefined surfaces and the part can be further stiffened by adding more fixed supports to the default fixturing. These fixed supports range from simple clamps to novel intelligent fixtures that enable the identification of critical process conditions, the compensation of error influences and the minimization of defective parts [Möhring 2016].

Vibrations during thin walled part machining are mainly related to the dynamics of the area of the part where the tool is interacting and the use of additional fixtures all around the part may require a large amount of clamping elements. Considering this fact, a mobile support system can be used to provide increased stiffness to the part precisely at the tool-part contact zone, following the tool around the part at the same velocity. Fei [Fei 2017, Fei 2018] introduces an ad hoc mobile support system attached to the ram of the machine tool to stiffen and damp the thin walled part while machining in order to suppress vibrations. In the literature the trend is to use robots as mobile supports for thin walled part machining, because of the additional flexibility and adaptability that robots provide. The robotic mobile support allows the suppression of vibrations and reduces deflections by improving the stiffness of the thin walled parts in the direction of the main vibration mode. Some authors focus their research on the coordination for dual-robot mirror milling system consisting of a machining hybrid robot, a supporting hybrid robot, and a fixture [Xiao 2018]. Other authors develop a force control solution in an industrial robot to be used in a milling machine in order to supress vibrations and deformation of the thin walled part [Esfandi 2017, Ozturk 2018].

A robot as mobile support provides a clear improvement in the dynamic response of the thin walled part, which has been demonstrated. Therefore, it is of high interest to predict the dynamic response of the combined robot and part system in order to simulate the dynamic response and stability of the machining operation. This machining capability prediction allows determining the viability of the robotic mobile support, saving time and costs compared to the experimental verification that requires physically moving the robot to the machining workshop.

A range of techniques has been developed to help dynamic design and vibration analysis of complex structures or systems. These techniques represent the structure through models so that the dynamic properties of the structure can be studied [Urgueira 1989].

These models can be broadly categorized as:

- Spatial Models. Analytical models based on the mass, stiffness and damping matrices representing physical properties of the Degrees of Freedom (DoF) of the 
structure, often obtained from finite element modelling (FEM).

- Modal Models. Analytical models which are obtained by extracting dynamic properties from analytical or experimental Frequency Response Function (FRF) measurements such as natural frequencies, mode shapes and damping ratios.

- Response Models. Analytical or experimental models characterized by the ratio of output response of the system to an input force. This relationship is described by an FRF matrix. These models are often considered more promising for the representation of the physical model since they do not have any approximations due to idealization or incompleteness of the measurements.

Developing an analytical model of the assembled robot and part system is time consuming and therefore, expensive; and developing experimental FRFs of the complete system may not be possible. Hence, a substructure analysis method will be used for the analysis of each component and then apply a coupling technique to derive the dynamic response of the assembled system [Ferreira 1998]. Thus, dynamics of the part and the robot can independently be considered, even analytically or experimentally. Then, by applying a coupling technique, the stability of the assembled system can be checked.

For the proposed case study of a robot as a mobile support the Receptance Coupling for Subsystem Analysis (RCSA) technique will be used. This technique was introduced by Bishop [Bishop 1954], who determined that a complicated oscillatory system can be regarded as if it was built up from simpler subsystems which are coupled together at certain coordinates.

The RCSA technique has been widely used due to the simplicity of its mathematical formulation, such as in HSM stability simulation. HSM simulation, which is crucial for chatter prediction and avoidance, requires knowledge of the system dynamics at the tool centre point. In general, a separate set of FRF measurements are performed for each combination of tool, tool holder and spindle on a particular machining centre. Thus, it is suggested to make only a single experiment at the tool holder tip of the machine tool, and the dynamics of the holder is coupled with the analytically obtained tool dynamics, in order to obtain the FRF of the complete system. This semi-analytical mode can provide accurate results and may save considerable time in applications where only the conditions related to the cutting tool are changed [Schmitz 2000, Schmitz 2001].

Moreover, from these first works for HSM tool point simulation, several authors have developed new improvements in the RCSA technique: For example, Park [Park 2003], considers more DoF at the tool holder-tool joint, Schmitz [Schmitz 2006] includes the analysis of additional coupling coordinates, Mancisidor [Mancisidor 2011] calculates the fixed boundary dynamic behaviour of the tool, and Ertürk [Ertürk 2006] proposes analytical modelling of all components of spindle-holder-tool assembly and these models are coupled in order to obtain tool point FRF by using receptance coupling and structural modification methods.

Therefore, RCSA is a validated substructure analysis technique for the prediction of the dynamic response of assembled systems. Specially for HSM tool point simulation, the capability of the technique for incorporating more considerations in the formulation has been proven. Hence, in the present work, the RCSA technique will be expanded for the frequency response prediction for robot assisted machining, when a thin walled part is supported by a robot, in order to determine the stability of the assembled system.

The rest of the paper is organised as follows. A first approach of the RCSA is introduced in Section 2 and experimentally validated in Section 3. The RCSA method is expanded for the calculation of the direct and crossed FRFs of the assembled system in Section 4 and validated in Section 5. The extended RCSA is applied to the proposed use case in Section 6, including machining stability analysis. Finally, conclusions are detailed in Section 7.

\section{FIRST APPROACH OF THE RCSA FOR ROBOT ASSISTED MACHINING PREDICTION}

Robot assisted machining for thin walled parts, which is the study case considered in the present work, is illustrated in Fig. 1. The robot supports the thin walled part in the most flexible direction during machining while the tool cuts on the opposite side of the part. Thereby, the robot locally increases the stiffness of the part avoiding deformations and unintended vibrations. The objective is to derive the frequency response of the part in the coordinate of interest of the assembled system composed by the robot and the part by considering the frequency responses of each component, the robot and the thin walled part. The assembled system is assumed linked by a highly stiff union, which is generated by the preload force exerted by the robot on the part. In this first RCSA approach for robot assisted machining only the most flexible direction is considered.

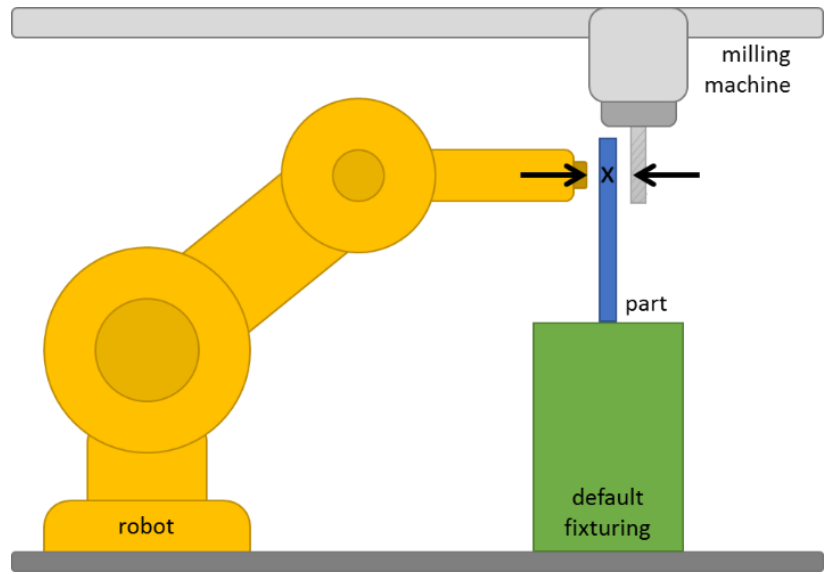

Fig. 1: Robot Assisted Machining for thin walled parts.

The unassembled system (Fig 2a.) is based on two separate components, the robot and the part, where two coordinates ( $\mathrm{R}$ and $\mathrm{P})$ will be connected by a highly stiff spring $\left(K_{x}\right)$ in the most flexible direction of the part ( $x$ axis). Looking at each component, the displacement $x$ in each coordinate can be written as:

$$
\begin{aligned}
& x_{P}=H_{x x P} f_{x P} \\
& x_{R}=H_{x x R} f_{x R}
\end{aligned}
$$

where, $f_{x P}$ and $f_{x R}$ are the input forces and $H_{x x P}$ and $H_{x x R}$ the FRFs in each point.

When considering the assembled system (Fig 2b), only the input force $F_{X P}$ is exerted in the part. As consequence of the union, both displacements $X_{p}$ and $X_{R}$ in the part and robot occur.

The equilibrium condition can be written as:

$$
F_{x P}=f_{x R}+f_{x P}
$$

The compatibility conditions are: 
$X_{R}=x_{R}$

$X_{P}=x_{P}$

In the assembled system, the only force exerted in the robot is coming from the stiff union. Therefore:

$f_{x R}=K_{x}\left(x_{P}-x_{R}\right)$
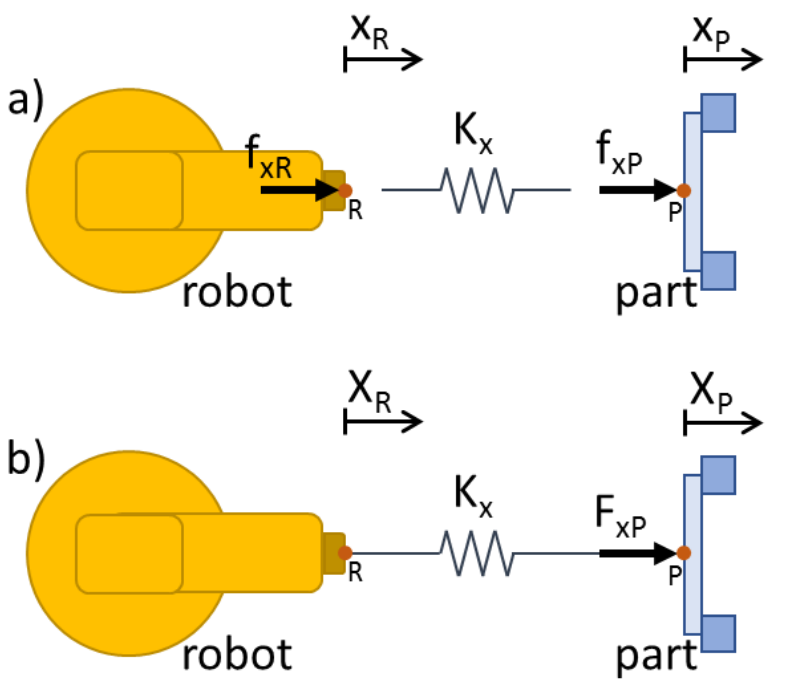

Fig. 2: a) Unassembled systems b) Assembled system.

Combining Eq. 1-5 the frequency response of the assembled system $G_{X x P}$ as the displacement of the part $X_{P}$ when an external force $F_{x p}$ is applied in the coordinate $\mathrm{P}$ is obtained (Eq. 6).

$$
\begin{gathered}
G_{x x P}=\frac{X_{P}}{F_{x P}}= \\
=H_{x x P}-H_{x x P}\left(H_{x x P}+H_{x x R}+\frac{1}{K_{x}}\right)^{-1} H_{x x P}
\end{gathered}
$$

\section{EXPERIMENTAL VALIDATION OF THE FIRST RCSA APPROACH}

The first approach of the RCSA technique for prediction of the frequency response of a thin walled part with a robot support has been experimentally validated. The FRFs of each component, the robot and the part, have been experimentally obtained. Then, the RCSA technique has been applied to calculate the resultant dynamic response. This response has been compared with the FRF obtained of the assembled system when the robot is exerting a certain force in the part in the most flexible direction ( $x$ axis).

\subsection{Experimental setup}

The robot is a Stäubli TX90L robot installed in a Soraluce FMT Multipurpose Milling Machine. The thin walled part is a C45J steel sheet with $450 \mathrm{~mm}$ length, $100 \mathrm{~mm}$ height and $6 \mathrm{~mm}$ width which is clamped at the ends to two rigid structures. Additionally, the part is risen with a rigid table for accessibility purpose (Fig. 3).

The FRFs are obtained with a data acquisition system. The displacement is measured with a PCB Piezotronics 352C68 accelerometer of $100 \mathrm{mV} / \mathrm{g}$ sensitivity and 0.5 to $10000 \mathrm{~Hz}$ range and the input force is exerted with an impact hammer 086D20 from PCB Piezotronics with $0.23 \mathrm{mV} / \mathrm{N}$ sensitivity.

For the robotic mobile support application, the robot is equipped with a ball caster as end effector, in order to exert a single directional force in the part, connected to a Kistler 9212 load cell to quantify the applied force. The load cell is screwed to the flange of the robot.

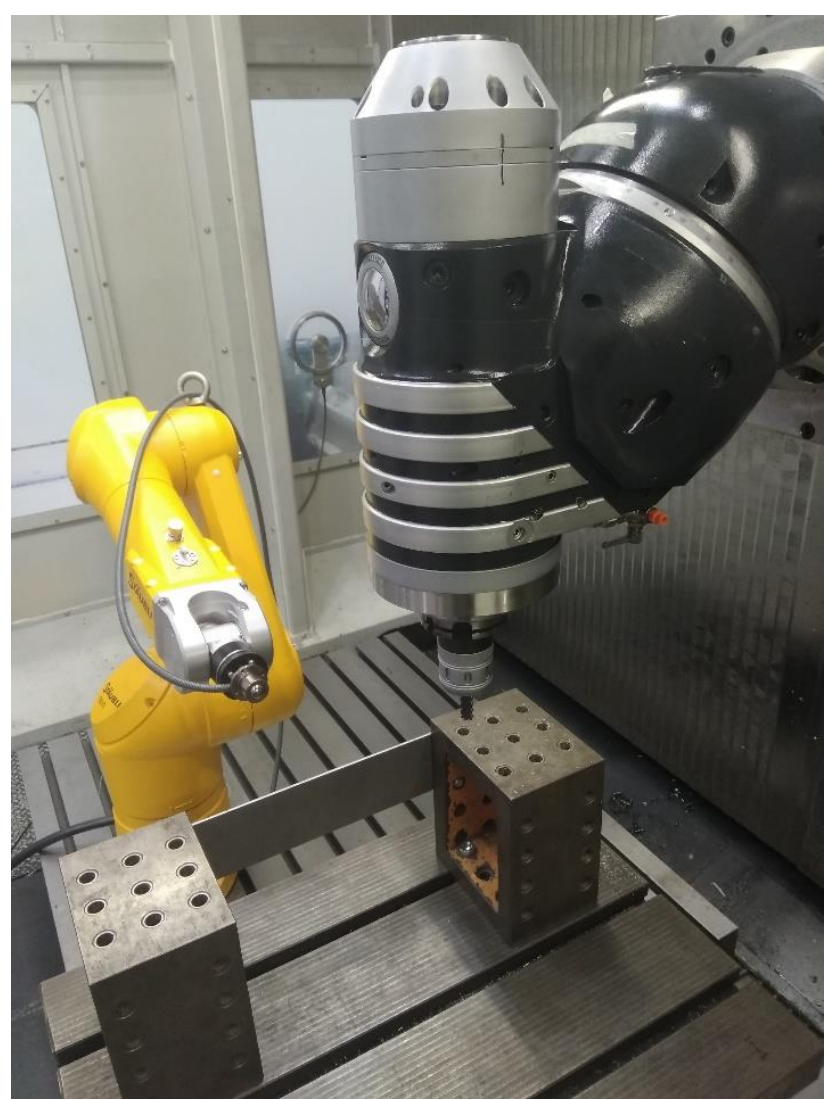

Fig. 3: Setup for the Robot Assisted Machining for thin walled parts, which is the study case.

\subsection{Experimental results}

In order to validate the RCSA for robot assisted machining the FRF of the robot in a certain pose $H_{x x R}$, the FRF of the part in the center point $H_{x x P}$ and the FRF of the assembled system $G_{x x P}$ are experimentally obtained. Then, RCSA technique derived in Eq. 6 is applied to the FRFs of the robot $H_{x x R}$ and the part $H_{x x P}$ to obtain a semi-analytical FRF of the assembled system. The connection stiffness $K_{x}$ value is assumed infinite, in order to simulate that the exerted preload of the robot is a perfect union between the robot and the part.

$$
K_{x} \rightarrow \infty
$$

Results are illustrated in Fig. 4. The FRF of the part $H_{x x P}$ shows that the part is very flexible with two main modes at $212 \mathrm{~Hz}$ and $567 \mathrm{~Hz}$. Besides, the FRF of the robot $H_{x x R}$ shows a stiffer response with a significant mode at $29 \mathrm{~Hz}$.

Both experimental and semi-analytical FRFs of the assembled system $G_{x x P}$ are very similar, validating the proposed first approach of RCSA for robot assisted machining frequency response prediction. Also, the obtained FRFs validate the effectiveness of the robot as mobile support, since the amplitude of the flexible modes of the part is highly reduced. In Fig. 4 it can be observed that the two main modes of the part have been almost totally suppressed. Experimentally a reduction in the amplitude of $99.96 \%$ at the frequency of the first mode and $99.87 \%$ at the second mode is achieved. The RCSA technique predicts an amplitude reduction of the $99.96 \%$ at the frequency of the first mode and $99.94 \%$ at the second one. 


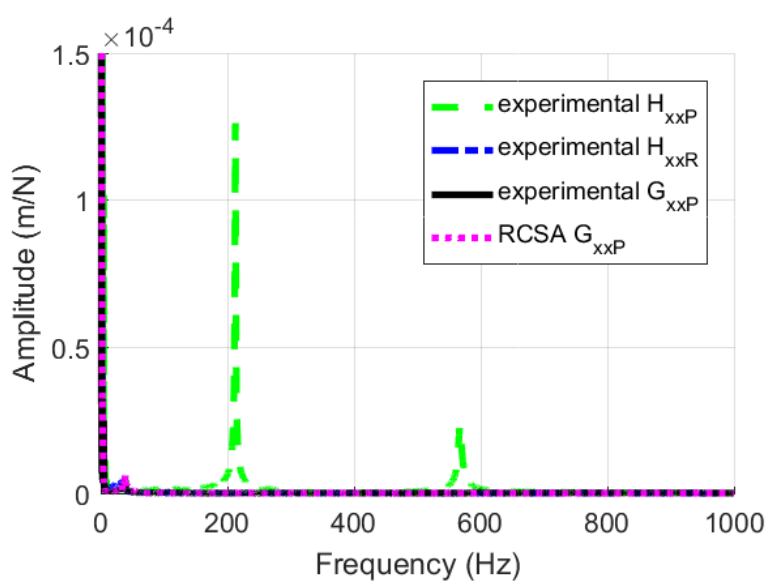

Fig. 4: Experimental FRFs and prediction with the RCSA technique.

For a more detailed comparison of experimental and semianalytical results, low frequency zone of Fig. 4 is zoomedin and shown in Fig. 5. At low frequencies, the assembled system presents its main mode at $38 \mathrm{~Hz}$. The RCSA technique has been able to properly predict the frequency of this mode with a $0.25 \%$ error.

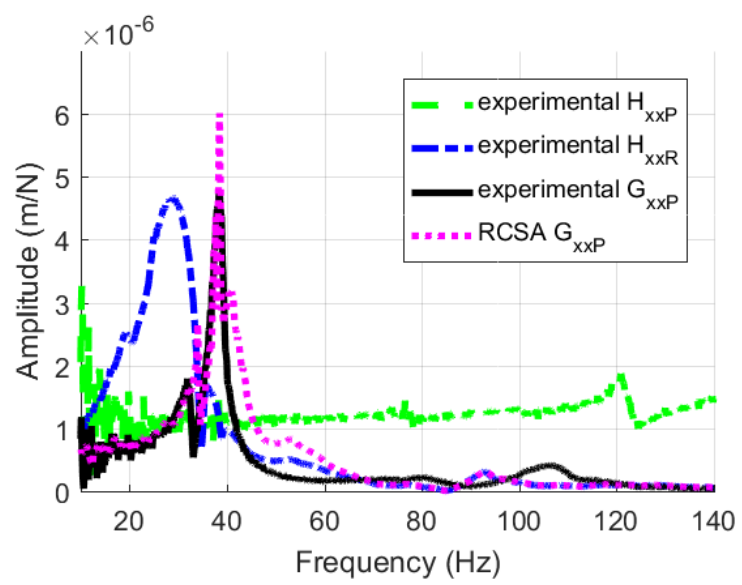

Fig. 5: Experimental FRFs and prediction with the RCSA technique at low frequencies.

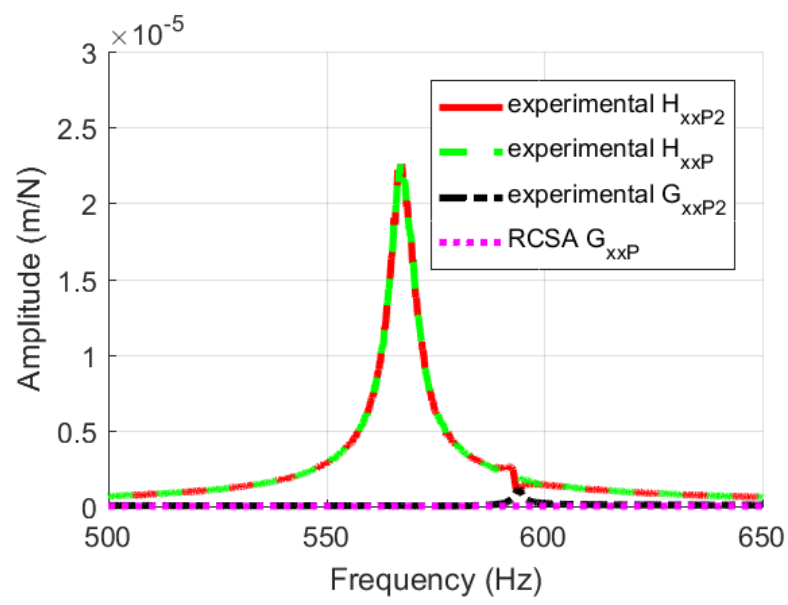

Fig. 6: FRFs around the second mode of the part.

Another zone of Fig. 4 to be analysed is around the second mode of the part (Fig 6). A discrepancy can be observed between the experimental assembled system and the RCSA prediction at $594 \mathrm{~Hz}$. This small difference may occur since the measuring accelerometer is not precisely located in the $\mathrm{P}$ coordinate but displaced $20 \mathrm{~mm}$ in the $y$ direction
(P2 coordinate), due to the ball caster at the robot tip making difficult the access to coordinate $P$.

\section{EXTENDED RCSA FOR ROBOT ASSISTED MACHINING PREDICTION}

In Section 2 a first introduction of the RCSA technique for robot assisted machining prediction is introduced. In this approach only the resultant frequency response in the most flexible direction is obtained. However, for a proper stability analysis not only the FRF in this axis is needed, but all direct and crossed FRFs of the assembled system.
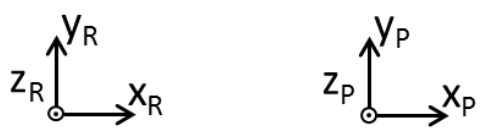

a)
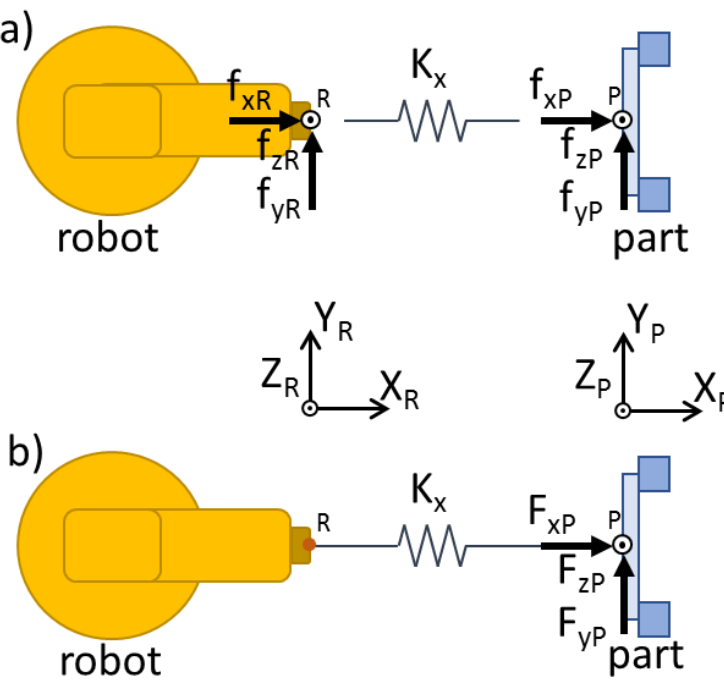

Fig. 7: a) Unassembled systems b) Assembled system in three axes.

Therefore, the unassembled system (Fig. 7a) is again based on two separate components, the robot and the part, where two coordinates $(R$ and $P$ ) will be connected by a stiff union $\left(K_{x}\right)$ in the $x$ axis but will be free, not connected, in the $y$ and $z$ axis. Nonetheless now displacements in three directions for the robot $\left(x_{R}, y_{R}, z_{R}\right)$ and the part $\left(x_{P}, y_{P}, z_{P}\right)$ are considered and can be written as function of the direct $\left(H_{x x}, H_{y y}, H_{z z}\right)$ and crossed $\left(H_{x y}, H_{x z}, H_{y x}, H_{y z}, H_{z x}, H_{z y}\right)$ FRFs and the input forces in the three directions $\left(f_{x} . f_{y} . f_{z}\right)$ for each component.

$\left[\begin{array}{l}x_{P} \\ y_{P} \\ z_{P}\end{array}\right]=\left[\begin{array}{lll}H_{x x P} & H_{x y P} & H_{x z P} \\ H_{y x P} & H_{y y P} & H_{y z P} \\ H_{z x P} & H_{z y P} & H_{z z P}\end{array}\right]\left[\begin{array}{l}f_{x P} \\ f_{y P} \\ f_{z P}\end{array}\right]$
$\left[\begin{array}{l}x_{R} \\ y_{R} \\ z_{R}\end{array}\right]=\left[\begin{array}{lll}H_{x x R} & H_{x y R} & H_{x z R} \\ H_{y x R} & H_{y y R} & H_{y z R} \\ H_{z x R} & H_{z y R} & H_{z z R}\end{array}\right]\left[\begin{array}{l}f_{x R} \\ f_{y R} \\ f_{z R}\end{array}\right]$

In the assembled system (Fig $7 \mathrm{~b})$, only the input forces $\left(F_{X P}\right.$ $\left.F_{y P}, F_{z P}\right)$ exerted in the part are considered, in the $\mathrm{P}$ coordinate of interest.

Equilibrium conditions can be written as:

$$
\begin{aligned}
& F_{x P}=f_{x R}+f_{x P} \\
& F_{y P}=f_{y P} \text { and } f_{y R}=0 \\
& F_{z P}=f_{z P} \text { and } f_{z R}=0
\end{aligned}
$$


As $y$ and $z$ directions are free, forces $f_{y R}$ and $f_{z R}$ in the robot are null and as $x$ direction is connected by a stiff union $K_{x}$, $f_{x R}$ is obtained by:

$f_{x R}=K_{x}\left(x_{P}-x_{R}\right)$

The compatibility conditions are:

$X_{R}=x_{R} \quad Y_{R}=y_{R} \quad Z_{R}=z_{R}$

$X_{P}=x_{P} \quad Y_{P}=y_{P} \quad Z_{P}=z_{P}$

Combining Eq. 7-11 and considering that when applying force in the $x$ direction on the assembled system $F_{X P}$, the forces in the other directions are null $F_{y P}=0$ and $F_{z P}=0$.

$G_{x x P}=H_{x x P}-H_{x x P}\left(H_{x x P}+H_{x x R}+\frac{1}{K_{x}}\right)^{-1} H_{x x P}$

$G_{y x P}=H_{y x P}-H_{y x P}\left(H_{x x P}+H_{x x R}+\frac{1}{K_{x}}\right)^{-1} H_{x x P}$

$G_{z x P}=H_{z x P}-H_{z x P}\left(H_{x x P}+H_{x x R}+\frac{1}{K_{x}}\right)^{-1} H_{x x P}$

Again, when applying force in the $y$ direction on the assembled system $F_{y P}$, the forces in the other directions are null $F_{X P}=0$ and $F_{z P}=0$.

$$
\begin{aligned}
& G_{x y P}=H_{x y P}-H_{x x P}\left(H_{x x P}+H_{x x R}+\frac{1}{K_{x}}\right)^{-1} H_{x y P} \\
& G_{y y P}=H_{y y P}-H_{y x P}\left(H_{x x P}+H_{x x R}+\frac{1}{K_{x}}\right)^{-1} H_{x y P} \\
& G_{z y P}=H_{z y P}-H_{z x P}\left(H_{x x P}+H_{x x R}+\frac{1}{K_{x}}\right)^{-1} H_{x y P}
\end{aligned}
$$

Finally, when applying force in the $z$ direction on the assembled system $F_{Z P}$, the forces in the other directions are null $F_{x P}=0$ and $F_{y P}=0$.

$$
\begin{aligned}
& G_{x z P}=H_{x z P}-H_{x x P}\left(H_{x x P}+H_{x x R}+\frac{1}{K_{x}}\right)^{-1} H_{x z P} \\
& G_{y z P}=H_{y z P}-H_{y x P}\left(H_{x x P}+H_{x x R}+\frac{1}{K_{x}}\right)^{-1} H_{x z P} \\
& G_{z z P}=H_{z z P}-H_{z x P}\left(H_{x x P}+H_{x x R}+\frac{1}{K_{x}}\right)^{-1} H_{x z P}
\end{aligned}
$$

where,

$G_{x x P}=\frac{X_{P}}{F_{x P}} \quad G_{x y P}=\frac{X_{P}}{F_{y P}} \quad G_{x z P}=\frac{X_{P}}{F_{z P}}$

$G_{y x P}=\frac{Y_{P}}{F_{x P}} \quad G_{y y P}=\frac{Y_{P}}{F_{y P}} \quad G_{y z P}=\frac{Y_{P}}{F_{z P}}$

$G_{z x P}=\frac{Z_{P}}{F_{x P}} \quad G_{z y P}=\frac{Z_{P}}{F_{y P}} \quad G_{z z P}=\frac{Z_{P}}{F_{z P}}$

Notice that in the proposed extended RCSA of Eq. 12-14, where only the $x$ direction is stiffly connected by $K_{x}$ and $y$ and $z$ are free, only the direct FRF of the robot in $x$ axis $H_{x x R}$ is required. Hence, Eq. 8 can be simplified to Eq. 2. Moreover, notice that the resulting FRF $G_{x x P}$ is the same as the one obtained in Eq. 6 which has been already validated.

The direct FRF of the robot in $x$ axis $H_{x x R}$ may be obtained experimentally for each position and orientation of the robot where it interacts with the part. In case of large parts that require multiple robot poses to support the part it may be interesting to use an elastic joint robot model to obtain the robot dynamics as proposed by Spong [Spong 1987] with joint stiffness data experimentally obtained according to the procedure proposed by Dumas [Dumas 2011]. 

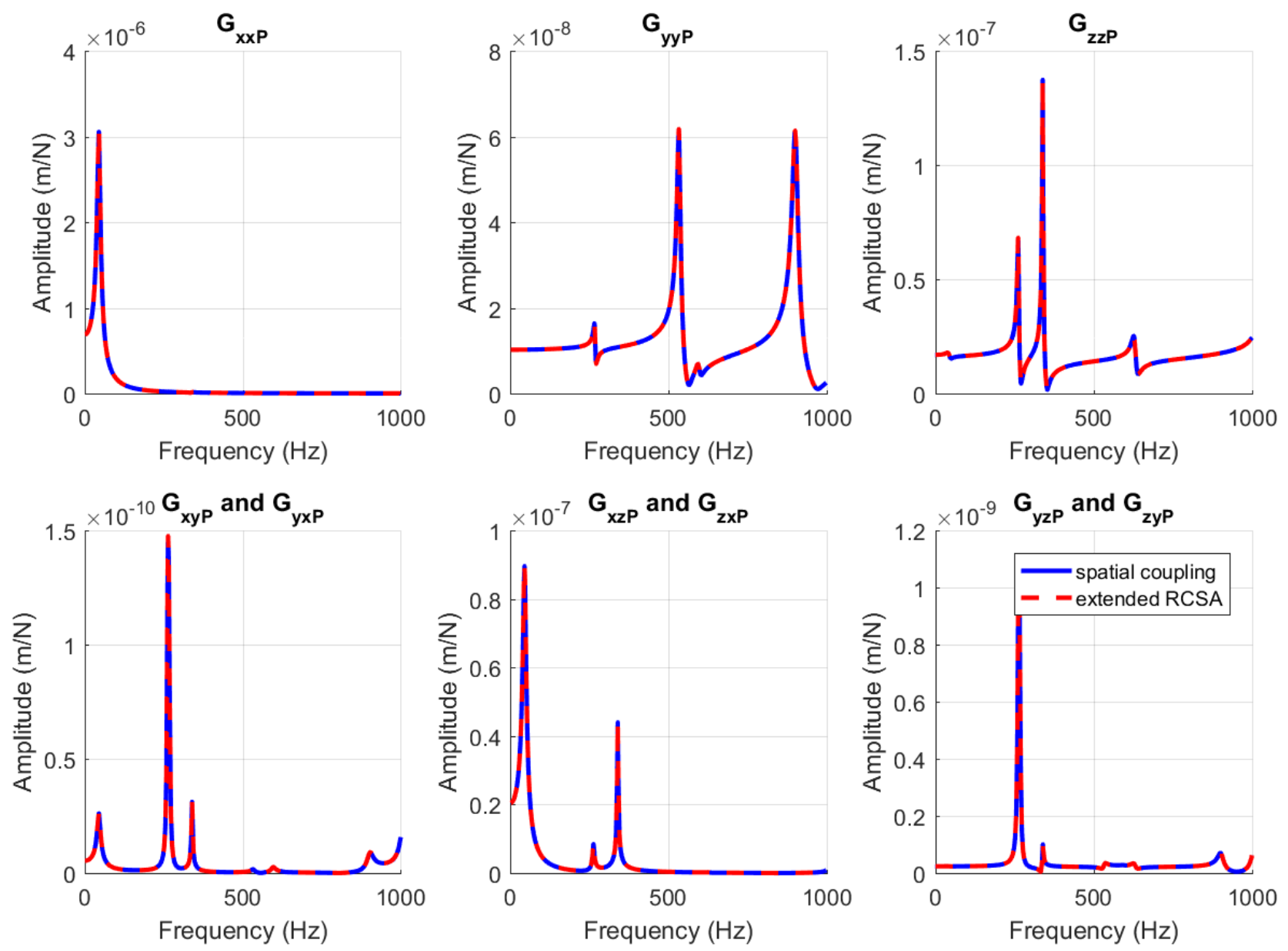

Fig. 8: Direct and crossed FRFs of the analytical validation.

$\left[M_{P}\right]_{n x n}\left[\ddot{x}_{P}\right]_{n x 1}+\left[C_{P}\right]_{n x n}\left[\dot{x}_{P}\right]_{n x 1}+\left[K_{P}\right]_{n x n}\left[x_{P}\right]_{n x 1}=\left[f_{P}\right]_{n x 1}$

$\left[M_{R}\right]_{1 x 1}\left[\ddot{x}_{R}\right]_{1 x 1}+\left[C_{R}\right]_{1 x 1}\left[\dot{x}_{R}\right]_{1 x 1}+\left[K_{R}\right]_{1 x 1}\left[x_{R}\right]_{1 x 1}=\left[f_{R}\right]_{1 x 1}$

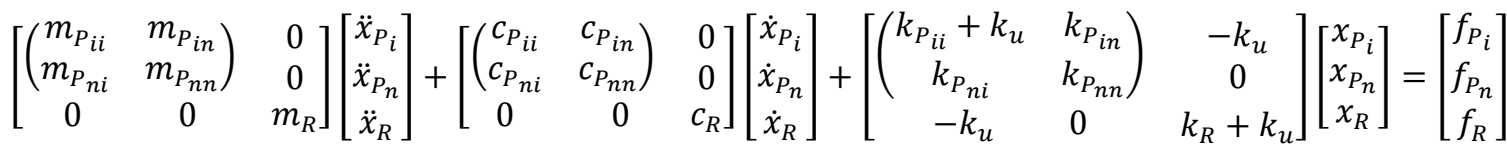

\section{VALIDATION OF THE EXTENDED RCSA APPROACH}

The extended approach of the RCSA for robot assisted machining prediction has been analytically validated. Unlike the first RCSA technique introduced in Section 2 that only needs the FRFs of the part in the $x$ direction, the extended RCSA technique uses direct and crossed FRFs of the part in the $\mathrm{P}$ coordinate, as shown in Eq. 11-19. Experimentally obtaining FRFs in $y$ and $z$ direction in a flat thin walled part may be tricky, thus an analytical validation is proposed.
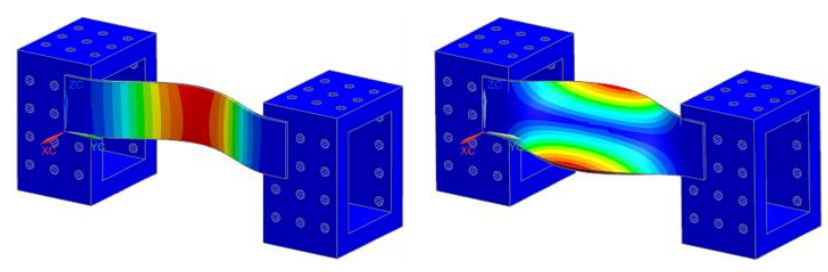

Fig. 9: FEM Model with the fitting of the two main modes, which are in $x$ axis.
On one hand, the part is modelled with Finite Element Method (FEM) verifying that the main modes of the model match the modes of the real part (Fig. 9). Then, CraigBampton method [Craig 1968] is applied to obtain a spatial model with $n$ DoF represented by the square $n \times n$ matrix of mass $M_{P}$ and stiffness $K_{P}$ while the damping matrix $C_{P}$ is generated based on experimental FRFs (Eq. 16). It is of high interest to obtain a precise model of the part, because it will be used later for the experimental prediction and stability analysis.

On the other hand, the robot is simplified to a second order model, considering the experimentally obtained main mode of the robot at $29 \mathrm{~Hz}$. Therefore, the model of the robot is composed by only a component of mass $M_{R}$, damping $C_{R}$ and stiffness $K_{R}$ (Eq. 17).

Once the spatial models of both the part and the robot are obtained the model of the coupled system is developed by a spatial coupling method, as shown in Eq. 18. Both systems are connected by a spring $K_{u}$ between the $i$ DoF of the part and the DoF of the robot.

From the spatial models of the unassembled components, the robot and the part, the required FRFs for the extended 
RCSA technique validation are calculated. A comparison of the obtained responses for both coupling techniques is illustrated in Fig 8, where the error between both techniques
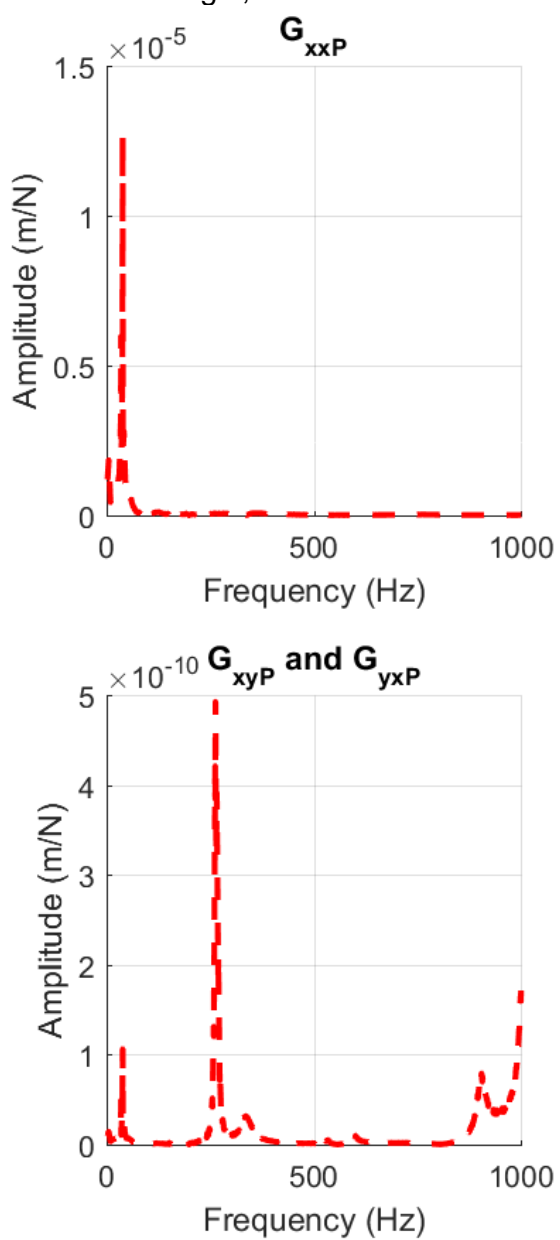

is negligible. Therefore, the equations for the extended RCSA technique are validated.
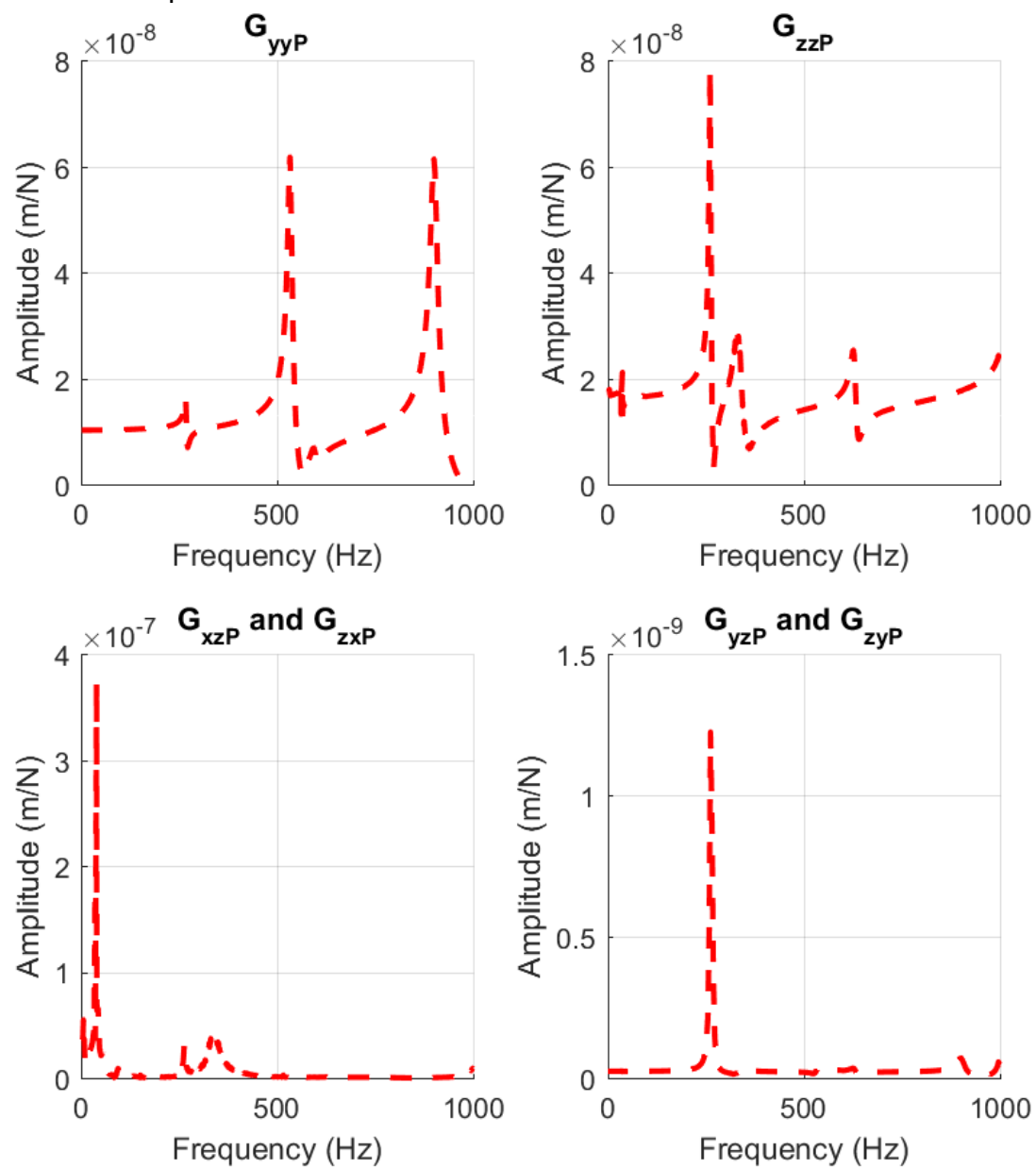

Fig. 10: Direct and crossed FRFs of the experimental validation.

\section{PREDICTION WITH THE EXTENDED RCSA APPROACH AND STABILITY ANALYSIS}

Once the extended RCSA technique is analytically validated an experimental prediction of the frequency response of the assembled system is calculated for milling stability analysis using the semi-discretization method proposed by Insperger [Insperger 2003].

For the prediction of the assembled robot and part system the experimental FRF of the robot from Section 3 is used. Besides, the analytical FRFs of the part are derived from its FEM, presented in Section 5, which matches well the modes of the real part.

Prediction of the frequency response with the extended RCSA approach is shown in (Fig 10). Even if the main effect of the robotic support is appreciated in the $x$ axis, responses in $y$ and $z$ axis are also affected and therefore the stability analysis too.

The direct and crossed FRFs of the part $\left(H_{x x P}, H_{y y P}, H_{z z P}\right.$ $\left.H_{x y P}, H_{x z P}, H_{y x P}, H_{y z P}, H_{z x P}, H_{z y P}\right)$ and of the assembled robot and part system calculated with the extended RCSA approach $\left(G_{x x P}, G_{y y P}, G_{z z P} G_{x y P}, G_{x z P}, G_{y x p}, G_{y z}, G_{z x P}, G_{z y P}\right)$ have been used to calculate the stability lobe diagram (Fig. 11) for machining capability analysis.

The cutting tool is a carbide 6 teeth and $16 \mathrm{~mm}$ diameter end mill, and main cutting conditions are a radial depth of cut of $0.2 \mathrm{~mm}$, a feed of $1800 \mathrm{~mm} / \mathrm{min}$ and a spindle speed of $1900 \mathrm{rpm}$.
Generally, without robotic mobile support the system is highly unstable, with a maximum depth of cut capacity at $1871 \mathrm{rpm}$ of $6.9 \mathrm{~mm}$. Instead, with robotic support the cutting is very stable from low spindle speed values. For the proposed cutting conditions for example, without robot support the maximum depth of cut applicable is $1.7 \mathrm{~mm}$ while with robotic support is $20 \mathrm{~mm}$, limited by the length of the tool.

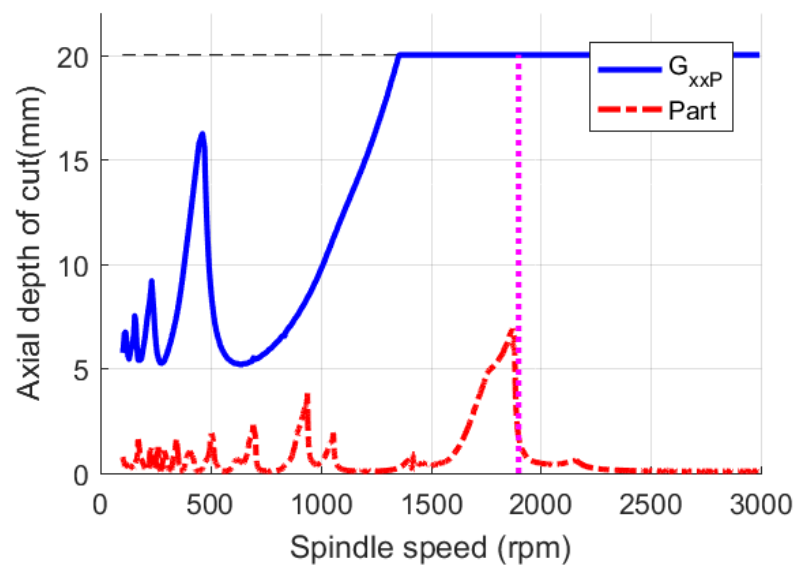

Fig. 11: Stability Lobe diagram.

\section{CONCLUSIONS}

The present work introduces a frequency response prediction technique for robot assisted machining based on 
experimental and/or analytical information for robot assisted machining use cases.

Robot assisted machining for thin walled parts is a promising technique to supress vibrations and deformations during the process. Therefore, it is of high interest to predict the stability of the assembled system, when the robot is exerting a certain force in the part.

Traditionally, the RCSA technique has been used in HSM for tool centre point dynamics prediction. In the present work, this technique has been extended to analyse the response in the three directions $(x, y, z)$ when a stiff union only in the $x$ axis is applied.

This technique has been validated, first in one axis experimentally and secondly analytically in the three axes. Experimentally, the FRFs of the robot, the part and the assembled systems in the direction corresponding to the main mode are acquire. Then the RCSA technique is used to predict the response and compare it to the experimenta one. The proposed extended RCSA technique is validated analytically by coupling spatial models of the robot and the part.

Finally, the stability analysis with a lobe diagram is shown for the part and the part with the robotic mobile support prediction. A significant improvement in the machining capability is predicted with a stability lobe diagram

\section{ACKNOWLEDGMENTS}

The authors would like to acknowledge the support provided by the European Commission under the Horizon 2020 Programme to the COROMA project under grant agreement no. 723853 .

\section{REFERENCES}

[Altintas. 2012] Altintas, Y. Manufacturing automation: metal cutting mechanics, machine tool vibrations, and CNC design. Cambridge university press, 2012.

[Bishop 1954] Bishop, R.E.D. The analysis and synthesis of vibrating systems. The Aeronautical Journal, 1954, Vol. 58 , No. 526, pp 703-719.

[Craig 1968] Craig, R., and Bampton, M. Coupling of substructures for dynamic analyses. AIAA journal, 1968, Vol. 6, No 7, pp. 1313-1319.

[Dumas 2011] Dumas, C., et al. Joint stiffness identification of six-revolute industrial serial robots. Robotics and Computer-Integrated Manufacturing, 2011, Vol.27, No. 4 pp. 881-888.

[Ertürk 2006] Ertürk, A., et al. k, E. Analytical modeling of spindle-tool dynamics on machine tools using Timoshenko beam model and receptance coupling for the prediction of tool point FRF. International Journal of Machine Tools and Manufacture, 2006, Vol.46, No.15, pp 1901-1912.

[Esfandi 2017] Esfandi, N., and Tsao, T. C. Robot assisted machining of thin-walled structures. IFACPapersOnLine, 2017, Vol.50, No.1, pp 14594-14599.
[Fei 2017] Fei, J., et al. Chatter mitigation using moving damper. Journal of Sound and Vibration, 2017, Vol.410, pp 49-63.

[Fei 2018] Fei, J., et al. Investigation of moving fixture on deformation suppression during milling process of thinwalled structures. Journal of Manufacturing Processes, 2018, Vol.32, pp 403-411.

[Ferreira 1998] Ferreira, J.V. Dynamic response analysis of structures with nonlinear components. Doctoral dissertation, Imperial College London, University of London, 1998.

[Insperger 2003] Insperger, T. et al. Stability of up-milling and down-milling, part 1: alternative analytical methods. International Journal of Machine Tools and Manufacture, 2003, Vol.43, No. 1, pp. 25-34

[Mancisidor 2011] Mancisidor, I., et al. Fixed boundaries receptance coupling substructure analysis for tool point dynamics prediction. Advanced Materials Research, Vol. 223, Trans Tech Publications, 2011

[Möhring 2016] Möhring, H.C., and Wiederkehr, P. Intelligent fixtures for high performance machining. Procedia CIRP, 2016, Vol.46, pp 383-390.

[Ozturk 2018] Ozturk, E., et al. Robotic assisted milling for increased productivity. CIRP Annals, 2018, Vol.67, No.1, pp 427-430.

[Park 2003] Park, S.S., et al. Receptance coupling for end mills. International Journal of Machine Tools and Manufacture, 2003, Vol.43, No.9, pp 889-896.

[Pricewaterhouse 2014] Pricewaterhouse Coopers, LLP. The new hire: how a new generation of robots is transforming manufacturing. Delaware Limited Liability Partnership, 2014

[Schmitz 2000] Schmitz, T.L., and Donalson, R.R. Predicting high-speed machining dynamics by substructure analysis. Cirp Annals, 2000, Vol.49, No.1, pp 303-308.

[Schmitz 2001] Schmitz, T.L., et al. Tool point frequency response prediction for high-speed machining by RCSA. Journal of Manufacturing Science and Engineering, 2001, Vol.123, No.4, pp 700-707.

[Schmitz 2006] Schmitz, T.L., and Duncan, G.S. Threecomponent receptance coupling substructure analysis for tool point dynamics prediction. Journal of Manufacturing Science and Engineering, 2006, Vol. 127, No. 4, pp 781790.

[Spong 1987] Spong, M. W. Modeling and Control of Elastic Joint Robots. Journal of Dynamics Systems, Measurement and Control, 1987, Vol.109, No. 4, pp. 310-318.

[Urgueira 1989] Urgueira, A.P.V. Dynamic analysis of coupled structures using experimental data. Doctoral dissertation, Imperial College London, University of London,1989.

[Xiao 2018] Xiao, J. L., et al. Research on the collaborative machining method for dual-robot mirror milling. The International Journal of Advanced Manufacturing Technology, 2018, pp 1-14. 